OPEN ACCESS

Edited by:

Linden $\mathrm{Hu}$,

Tufts University, USA

Reviewed by:

Janakiram Seshu,

University of Texas at San Antonio,

USA

Robert D. Gilmore,

Centers for Disease Control and

Prevention, USA

Job E. Lopez,

Baylor College of Medicine, USA

*Correspondence:

$X$. Frank Yang

xfyang@iupui.edu

Yongliang Lou

|y|@wmu.edu.cn

${ }^{\dagger}$ Co-first authors.

Received: 27 January 2017 Accepted: 30 March 2017

Published: 20 April 2017

Citation:

Xiang X, Yang Y, Du J, Lin T, Chen T, Yang XF and Lou Y (2017)

Investigation of ospC Expression Variation among Borrelia burgdorferi Strains.

Front. Cell. Infect. Microbiol. 7:131. doi: 10.3389/fcimb.2017.00131

\section{Investigation of ospC Expression Variation among Borrelia burgdorferi Strains}

\author{
Xuwu Xiang ${ }^{1,2+}$, Youyun Yang ${ }^{2 \dagger}$, Jimei Du ${ }^{1}$, Tianyu Lin $^{3}$, Tong Chen ${ }^{1,2}$, X. Frank Yang ${ }^{1,2 *}$ \\ and Yongliang Lou ${ }^{1 *}$
}

${ }^{1}$ Key Laboratory of Laboratory Medicine, Ministry of Education of China, School of Laboratory Medicine, Wenzhou Medical University, Wenzhou, China, ${ }^{2}$ Department of Microbiology and Immunology, Indiana University School of Medicine, Indianapolis, IN, USA, ${ }^{3}$ College of Arts and Sciences, University of Pennsylvania, Philadelphia, PA, USA

Outer surface protein $\mathrm{C}(\mathrm{OspC})$ is the most studied major virulence factor of Borrelia burgdorferi, the causative agent of Lyme disease. The level of OspC varies dramatically among $B$. burgdorferi strains when cultured in vitro, but little is known about what causes such variation. It has been proposed that the difference in endogenous plasmid contents among strains contribute to variation in OspC phenotype, as B. burgdorferi contains more than 21 endogenous linear (Ip) and circular plasmids (cp), and some of which are prone to be lost. In this study, we analyzed several clones isolated from B. burgdorferi strain 297, one of the most commonly used strains for studying ospC expression. By taking advantage of recently published plasmid sequence of strain 297, we developed a multiplex PCR method specifically for rapid plasmid profiling of $B$. burgdorferi strain 297. We found that some commonly used 297 clones that were thought having a complete plasmid profile, actually lacked some endogenous plasmids. Importantly, the result showed that the difference in plasmid profiles did not contribute to the ospC expression variation among the clones. Furthermore, we found that $B$. burgdorferi clones expressed different levels of BosR, which in turn led to different levels of RpoS and subsequently, resulted in OspC level variation among $B$. burgdorferi strains.

Keywords: Borrelia burgdorferi, OspC, endogenous plasmid, multiplex PCR, BosR

\section{INTRODUCTION}

Lyme disease is the most common arthropod-born disease in the United States, Europe as well as Asia (Samuels, 2011; Radolf et al., 2012). The causative agent, Borrelia burgdorferi, is a spirochete that has a dual-membrane cell envelope. Unlike other Gram-negative bacteria that have lipopolysaccharide (LPS) on the outer membrane, B. burgdorferi outer membrane lacks LPS and contains numerous lipoproteins. These outer surface lipoproteins are differentially expressed and play key roles in host adaptation during its enzootic cycle between ticks and mammals (Radolf et al., 2012; Caimano et al., 2016). For example, the major outer surface lipoprotein C (OspC), is induced in B. burgdorferi during nymphal tick feeding and during early phase of mammalian infection, and it plays an essential role for spirochetes to establish early infection and may also be important for spirochetal transmission from tick to mammal (Grimm et al., 2004b; Pal et al., 2004; Carrasco et al., 2015a).

The genome of B. burgdorferi comprises of more than 21 linear and circular endogenous plasmids with 5-56 kb in size (Fraser et al., 1997; Casjens et al., 2000, 2012), making its genome 
have the largest plasmid content among any reported bacteria (Chaconas and Norris, 2013). Many endogenous plasmids encode outer surface lipoproteins, and propagation of spirochetes in vitro can lead to plasmid loss, which often results in attenuation of infection in mice or a defect in ticks (Purser and Norris, 2000). As such, a crucial step for genetic manipulation of $B$. burgdorferi is to perform plasmid profile for each clone to be sure the phenotype is not due to a plasmid loss. In addition, studies on the correlation between the loss of a particular plasmid and an in vitro or in vivo phenotype have led to identification of several virulence-associated genes (Purser et al., 2003; Revel et al., 2005). However, the contribution of each plasmid-encoded genes to the life cycle of $B$. burgdorferi remains largely unknown.

$B$. burgdorferi sensu lato (s.l.) complex includes B. burgdorferi sensu stricto (s.s.), B. afzelii, and B. garinii. B. afzelii, and B. garinii are found outside of the United States. B. burgdorferi s.s. are highly heterogeneous with significant variation in virulence and endogenous plasmid content. B. burgdorferi s.s. strain B31, which was isolated from a tick collected on Shelter Island (Burgdorfer et al., 1982), is the first sequenced strain and has been widely used for Lyme disease research. Another widely used B. burgdorferi strain is strain 297, which was isolated from the cerebral spinal fluid (CSF) sample of a Lyme borreliosis patient (Steere et al., 1983; Hughes et al., 1992). The endogenous plasmid sequence of B. burgdorferi strain 297 was published in 2011(Schutzer et al., 2011).

The advantage of using B. burgdorferi strain 297 to study gene regulation of $B$. burgdorferi is that $\operatorname{os} p C$ is highly expressed under the in vitro cultivation condition. Expression of ospC is induced by elevated temperature $\left(37^{\circ} \mathrm{C}\right.$ relative to ambient temperature, $\left.25^{\circ} \mathrm{C}\right)$, low $\mathrm{pH}(\mathrm{pH} 6.8-7.0)$, and high cell density (Yang et al., 2000). In fact, while high level of ospC expression can be achieved in B. burgdorferi strain B31 by temperature shift (first at $25^{\circ} \mathrm{C}$ then shift to $35^{\circ} \mathrm{C}$ ), osp C expression in strain 297 can be easily achieved by directly cultivating the spirochete at 35 or $37^{\circ} \mathrm{C}$ without temperature shift. The level of OspC in 297 strain can be readily visualized as one of the most dominant bands in the protein profile when separated on Coomassie stained gel. This advantage has contributed in part, to the discovery of the first regulatory pathway in B. burgdorferi, the Rrp2-RpoNRpoS pathway. In this pathway, the two-component system Hk2Rrp2 activates the $\sigma^{\mathrm{N}}-\sigma^{\mathrm{S}}$ (RpoN-RpoS) sigma factor cascade, leading to the production of $\sigma^{S}$ (RpoS), which in turn, controls expression of many virulence genes including ospC and many other genes (for review, see, Samuels, 2011; Radolf et al., 2012; Ouyang et al., 2014; Ye et al., 2016b). In addition, many other factors including BosR (Hyde et al., 2009; Ouyang et al., 2009, 2011), BadR (Miller et al., 2013; Ouyang and Zhou, 2015), DsrA (Lybecker and Samuels, 2007), Rrp1 (Rogers et al., 2009; Sze et al., 2013; He et al., 2014), BtmA (Troxell et al., 2013), SodA (Esteve-Gassent et al., 2015), BBD18 (Dulebohn et al., 2014), also influence $r p o S$ and ospC expressions.

Performing plasmid profile for strain 297 has been challenging, as the endogenous plasmid sequences for this strain were not available until recently. In the past, primers used for tracking most of the plasmids of strain 297 clones were designed based on the sequence of strain B31, which is not appropriate. As such, it is difficult to determine whether the parental low passage 297 strain is a single or mixed clones. Similarly, it is also difficult to determine whether the commonly used 297 clones derived from the original 297 strain have a complete plasmid content. In this study, based on published plasmid sequences of B. burgdorferi strain 297 (Schutzer et al., 2011; Casjens et al., 2012), we developed a multiplex PCR method specifically for rapid plasmid profiling of strain 297 . We analyzed plasmid profiles of the original 297 strain and the derived clones with different levels of ospC expression. Since it has been reported that genes carried on certain plasmids in strain B31, e.g., bbd18 on lp17, influence ospC expression (Sarkar et al., 2011; Dulebohn et al., 2014), we tested whether the difference in plasmid contents contributes to the variation in OspC levels among the clones. We found that different clones expressed different levels of BosR, and such difference, not the difference in their plasmid profiles, contributes to the ospC expression variation among B. burgdorferi strains.

\section{MATERIALS AND METHODS}

\section{B. burgdorferi Strains and Culture Conditions}

The parental B. burgdorferi strain 297 is a non-clonal strain, originally obtained from culturing the CSF sample of a Lyme borreliosis patient (Steere et al., 1983; Hughes et al., 1992). No more than three passages from the original strain was used in this study. AH130 and PL133 were derived clones from original strain 297. Spirochetes were cultivated in complete BarbourStoenner-Kelly-II (BSK-II) medium (Barbour, 1984) at $37^{\circ} \mathrm{C}$ with $5 \% \mathrm{CO}_{2}$. Semi-solid agar plating of B. burgdorferi was carried out as previously described [complete Barbour-Stoenner-KellyII (BSK-II) medium with 2.5\% agar] (Samuels, 1995). Types of colony morphology were observed 8-12 days after plating.

\section{Sodium Dodecyl Sulphate-Polyacrylamide Gel Electrophoresis (SDS-PAGE) and Immunoblotting}

The method for SDS-PAGE was described previously (Carrasco et al., 2015b). Briefly, spirochetes were cultured from $10^{4}$ cells $/ \mathrm{ml}$ and harvested at day 7 (stationary phase $10^{8}$ cells $/ \mathrm{ml}$ ) by centrifugation at $8,000 \mathrm{~g}$ for $10 \mathrm{~min}$ and washed two times with PBS ( $\mathrm{pH} 7.4$ ) at $4^{\circ} \mathrm{C}$. Pellets were suspended in SDS buffer containing $50 \mathrm{mM}$ Tris- $\mathrm{HCl}$ ( $\mathrm{pH} 8.0), 2 \%$ sodium dodecyl sulfate (SDS), and $10 \mathrm{mM}$ dithiothreitol (DTT). Cell lysates $\left(5 \times 10^{7}\right.$ cells per lane) were separated by $12 \%$ SDS-PAGE and stained with Coomassie blue or transferred to nitrocellulose membranes (GE-Healthcare, Milwaukee, WI). Membranes were blotted with monoclonal antibodies against FlaB, RpoS, and BosR (He et al., 2008; Xu et al., 2010; Troxell et al., 2013) with 1:1,000, 1:50, and 1:500 dilutions, respectively, and then with goat anti-mouse IgGHRP secondary antibody (1:1,000, Santa Cruz Biotechnology). Detection of horseradish peroxidase activity was determined using enhanced chemiluminescence method (Thermo pierce ECL Western Blotting Substrate), and subsequently by exposure to X-ray film. 


\section{Primer Design}

Multi-alignment among all 19 endogenous plasmid sequences of B. burgdorferi strain 297 (Table 1) were performed using ALIGNMENT tool in Software Vector NTI (Thermo Fisher Scientific, CA). The identified unique sequences of each primer pair for PCR of each plasmid were then subjected to BLAST analysis (National Center for Biotechnology Information) against all published Borrelia chromosomal sequences in order to confirm no potential homology with chromosomal sequence (note that B. burgdorferi strain 297 chromosomal sequence is not available). General guidelines for primer design were as follows: (a) Primers should be at least 20 nucleotides but not longer than 28 nucleotides, to minimize the possibility of primer-dimer formation and unspecific binding; (b) CG nucleotides should be distributed equally on both ends; (c) All PCR products should differ in size by at least $20 \mathrm{bp}$ in order to allow efficient separation by gel electrophoresis. See primer sequences in Tables 2, 3 .

\section{Multiplex PCR and Gel Electrophoresis}

PCR was performed by using TaKaRa Ex Taq kit (Clontech, USA). Twenty five microliters of optimized PCR reaction includes $100 \mathrm{ng}$ of boiled whole cell lysate of B. burgdorferi (as DNA template), $2.5 \mu \mathrm{l}$ of $10 \times$ Ex Taq Buffer, $2.5 \mu l$ of $10 \times$ dNTP Mixture, $1 \mu \mathrm{l}$ of Ex Taq DNA polymerase, and $2 \mu \mathrm{l}$ of $12.5 \times$ primer mix. Given that each primer pair has different amplification efficiency, the concentrations for each primer pair used in the primer mix were adjusted based on the PCR result. For the primer mix to amplify linear plasmids (mixLp), $222 \mu \mathrm{l}$ of $12.5 \times$ mixlp primer stock includes $12 \mu \mathrm{l}$ of $50 \mu \mathrm{M}$ primer stock for $\operatorname{lp} 25, \operatorname{lp} 54, \operatorname{lp} 28-4,15 \mu \mathrm{l}$ for $\operatorname{lp} 38$, and $10 \mu \mathrm{l}$ for $\operatorname{lp} 28-1$, lp28-3, lp28-5, lp28-6, lp17, lp36. For the primer mix to amplify circular plasmids (mixCp), $210 \mu \mathrm{l}$ of $12.5 \times$ mixcp primer stock includes $15 \mu \mathrm{l}$ of $50 \mu \mathrm{M}$ primer stock for cp32-3, $10 \mu \mathrm{l}$ for cp26, $10 \mu l$ for cp32-1, cp32-4, cp32-5, cp32-6, cp32-9, cp32-11, cp3212. The PCR reaction conditions were as the following: (1) 1 cycle of initial denaturation at $94^{\circ} \mathrm{C}$ for 5 mins; (2) 40 cycles of amplification including denaturation at $94^{\circ} \mathrm{C}$ for $30 \mathrm{~S}$, annealing at $54^{\circ} \mathrm{C}$ for $1 \mathrm{~min}$, and extension at $68^{\circ} \mathrm{C}$ for $1 \mathrm{~min}$; (3) 1 cycle of final extension at $68^{\circ} \mathrm{C}$ for $5 \mathrm{~min}$. PCR products were separated using 3\% metaphor agarose gel (Lonza, Cohasset MN, USA) in TBE buffer (0.089 M Tris base, $0.089 \mathrm{M}$ boric acid, $2 \mathrm{mM}$ EDTA, $\mathrm{pH} 8.2-8.4$ ) by running $100 \mathrm{~V}$ for $1 \mathrm{~h}$ and then $80 \mathrm{~V}$ for $2 \mathrm{~h}$.

\section{RESULTS}

\section{Establish a Multiplex PCR Method for Rapid Plasmid Profiling of $B$. burgdorferi Strain 297}

Genome sequence revealed that B. burgdorferi strain 297 contains at least 10 linear and 10 circular plasmids ranging from 17 to $54 \mathrm{~Kb}$. The published plasmid sequences for strain 297 include 19 plasmids (Schutzer et al., 2011; Casjens et al., 2012). The strain 297 used for sequencing lost linear plasmid, lp25, so lp25 sequence of strain 297 is not available. In B. burgdorferi strain $\mathrm{B} 31, \mathrm{lp} 25$ contains at least two genes $p n c A$ and $b p t A$, which are important for the enzootic cycle of B. burgdorferi (Purser et al.,
TABLE 1 | List of endogenous plasmids in B. burgdorferi strain 297.

\begin{tabular}{lllcc}
\hline $\begin{array}{l}\text { Linear or circular } \\
\text { plasmids }\end{array}$ & Designation & Gene bank & Size(bp) & CG(\%) \\
Ip17 & D & Gl: 410108990 & 12,965 & 24.1 \\
Ip25 & E & / & $/$ & $/$ \\
Ip28-1 & F & Gl: 410108985 & 20,978 & 30.7 \\
Ip28-3 & H & Gl: 410108988 & 25,211 & 25.0 \\
Ip28-4 & I & Gl: 410108991 & 25,788 & 24.5 \\
Ip28-5 & Y & Gl: 410108986 & 20,974 & 24.3 \\
Ip28-6 & Z & Gl: 410108984 & 22,135 & 33.0 \\
Ip36 & K & Gl: 410108983 & 22,715 & 25.7 \\
Ip38 & J & Gl: 410108989 & 27,035 & 25.4 \\
Ip54 & A & Gl: 256041893 & 48,220 & 28.3 \\
cp26 & B & Gl: 410108980 & 26,514 & 26.2 \\
cp32-1 & P & Gl: 410108993 & 30,902 & 29.2 \\
cp32-3 & S & Gl: 410108995 & 30,262 & 28.8 \\
cp32-4 & R & Gl: 410108981 & 30,301 & 29.2 \\
cp32-5 & V & Gl: 410108979 & 30,636 & 29.2 \\
cp32-6 & M & Gl: 410108976 & 30,641 & 29.1 \\
cp32-7 & O & Gl: 410108978 & 21,165 & 28.8 \\
cp32-9b & N & Gl: 410108975 & 21,172 & 28.3 \\
cp32-11 & W & Gl: 410108994 & 30,286 & 28.9 \\
cp32-12 & X & Gl: 410108974 & 30,795 & 29.3 \\
\hline & & & & \\
\hline
\end{tabular}

${ }^{a}$ The sequence of Ip25 of 297 strain is not available.

${ }^{b}$ cp32-7 and cp32-9 are previously named as cp18-1 and cp18-2, respectively.

2003; Grimm et al., 2004a; Revel et al., 2005). It was reported previously that strain 297 has lp25 (Grimm et al., 2004a; Revel et al., 2005). Of note, two previously reported circular plasmids, cp18-1 and cp18-2 in strain 297 (Caimano et al., 2000), were the result of $\sim 9 \mathrm{Kbp}$ truncation from $\mathrm{cp} 32$, and were subsequently renamed as cp32-7 and cp32-9 (Casjens et al., 2012).

All PCR primer pairs specific for each endogenous plasmid were designed based on multi-alignment of all published plasmid sequences of B. burgdorferi strain 297 (Schutzer et al., 2011), followed by BLAST analyses against published B. burgdorferi B31 chromosomal sequences (see Materials and Methods). Since the sequence of lp25 of strain 297 is not available, the primer pair for lp25 of strain 297 was designed based on the bptA gene sequence from lp25 of strain B31 (Revel et al., 2005). The specificity and efficiency of each primer pair for corresponding plasmid were confirmed by regular PCR (Figures 1A,B). Then, primer pairs for all linear plasmids or circular plasmids were mixed together (mixLp or mixCp), and two multiplex PCR reactions were performed accordingly. The sizes of PCR products in each of the two groups were designed to differ at least $20 \mathrm{bps}$, so that they could be readily separated by gel electrophoresis (Figure 1).

\section{Comparison of Plasmid Contents among B. burgdorferi Clones with Different OspC Levels}

Having established a rapid plasmid profiling method specific for strain 297, we sought to examine the plasmid contents of some commonly used clones of B. burgdorferi strain 297. Clone AH130 was isolated on semi-solid agar plate from the 
TABLE 2 | PCR primer sequences for each linear plasmid.

\begin{tabular}{|c|c|c|c|c|c|c|}
\hline Linear plasmid & Sequence $5^{\prime}->3^{\prime}$ & Length (bp) & Coordination $5^{\prime}-3^{\prime}$ & $\operatorname{Tm}\left({ }^{\circ} \mathrm{C}\right)$ & $\begin{array}{l}\text { Concentration in } 12.5 \\
\times \text { primer mix (uM) }\end{array}$ & Amplicon size (bp) \\
\hline Ip25F* & CGTTATCTACCGTTATAGGTTTGA & 25 & 266-290 & 52.5 & 2.7 & 100 \\
\hline Ip54F & TCTTAATATCAACCTAGAATATTCC & 25 & $965-989$ & 48.0 & 2.7 & 125 \\
\hline Ip54R & TAACAGACGAAGAAGAAGAGACTाT & 25 & $1,089-1,065$ & 53.7 & 2.7 & \\
\hline Ip28-6F & ATTGATGAATGGCGTTACCATTAGT & 25 & $11,486-11,510$ & 54.9 & 2.25 & 151 \\
\hline Ip28-4F & CTTGCGATCTACCAACAATGAGTAA & 25 & $27-51$ & 55.1 & 2.7 & 175 \\
\hline Ip28-4R & AGTTATCTGATATTAGGAGATAGT & 25 & $201-177$ & 48.5 & 2.7 & \\
\hline Ip28-1F & TCAATCAAACATATTGGGTGAAGAA & 25 & $53,00-5,324$ & 53.0 & 2.25 & 200 \\
\hline Ip28-1R & TTGCCTGTATTGCTAAATTACTATG & 25 & $5,499-5,475$ & 51.5 & 2.25 & \\
\hline Ip28-3F & TGTCTAAGGAAGGTTTAAGGCTTAT & 25 & $21,131-21,155$ & 53.8 & 2.25 & 225 \\
\hline Ip17R & CGACTTCTTATATAGCTGAGATTCT & 25 & $12,781-12,757$ & 51.7 & 2.25 & \\
\hline Ip38F & AATCCAGGTATTCTTGTTGCTGGTC & 25 & $20,896-20,920$ & 57.2 & 3.38 & 325 \\
\hline Ip38R & TTATTAGGAGACGATATTAATATAA & 25 & $21,220-21,196$ & 45.0 & 3.38 & \\
\hline Ip36F & AAGTGGTGAATTGGAGGAGCCTATT & 25 & $15,394-15,418$ & 58.2 & 2.25 & 369 \\
\hline Ip36R & TTAGCAAAGTTGTCAAGGCGTGTAGA & 26 & $15,762-15,737$ & 58.5 & 2.25 & \\
\hline
\end{tabular}

${ }^{*}$ The primer sequences for 1025 were based on bptA gene sequence.

TABLE 3 | PCR primer sequences for each circular plasmid.

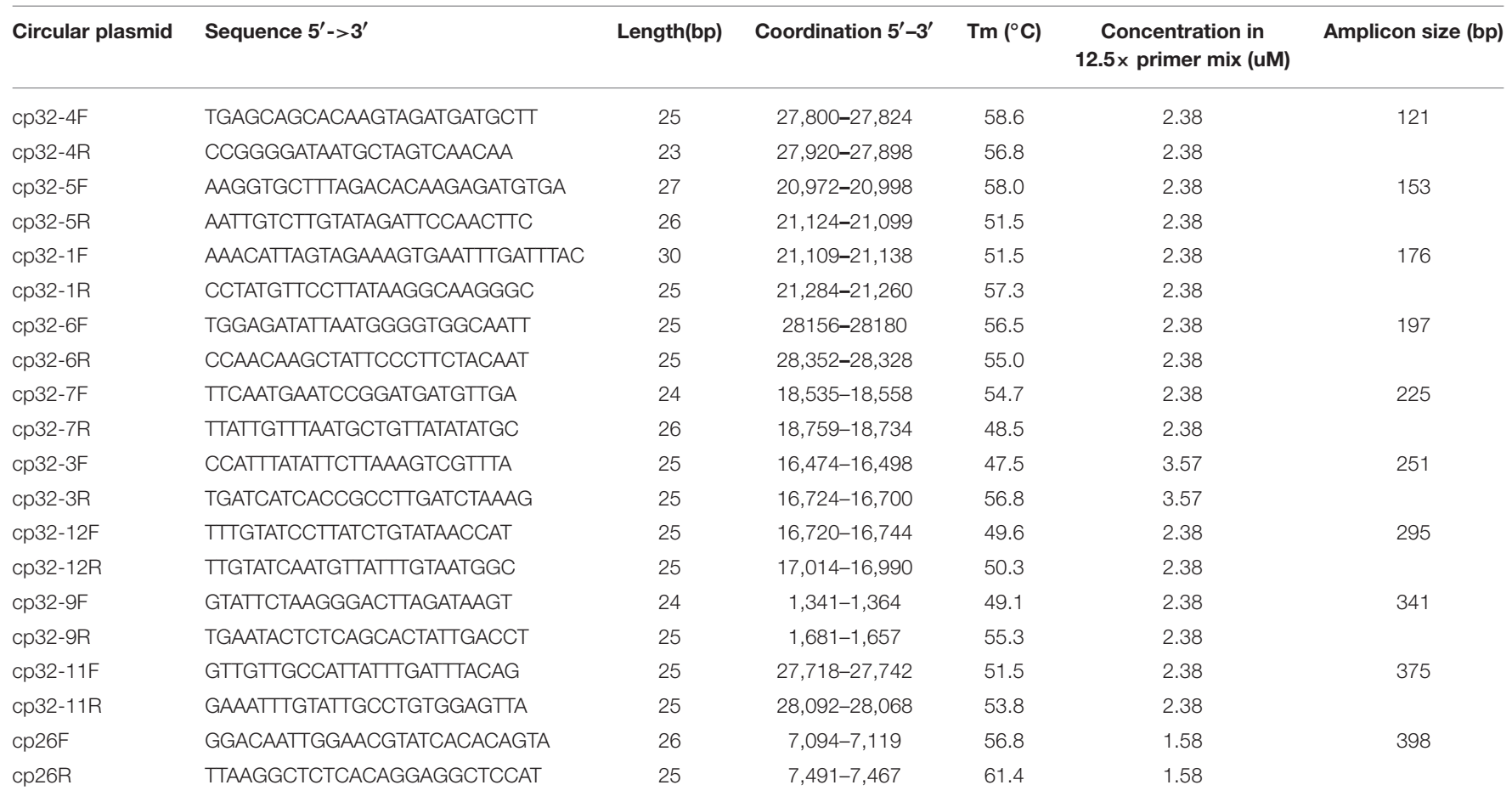

parental low passage B. burgdorferi strain 297 (Yang et al., 2004). Clone PL133 was isolated from mice after repeated rounds of needle inoculation by culturing ear-punch biopsies (Revel et al.,
2005; Blevins et al., 2007). Although both AH130 and PL133 are infectious in mice, they produced different levels of OspC under the in vitro cultivation conditions: AH130 produces very 

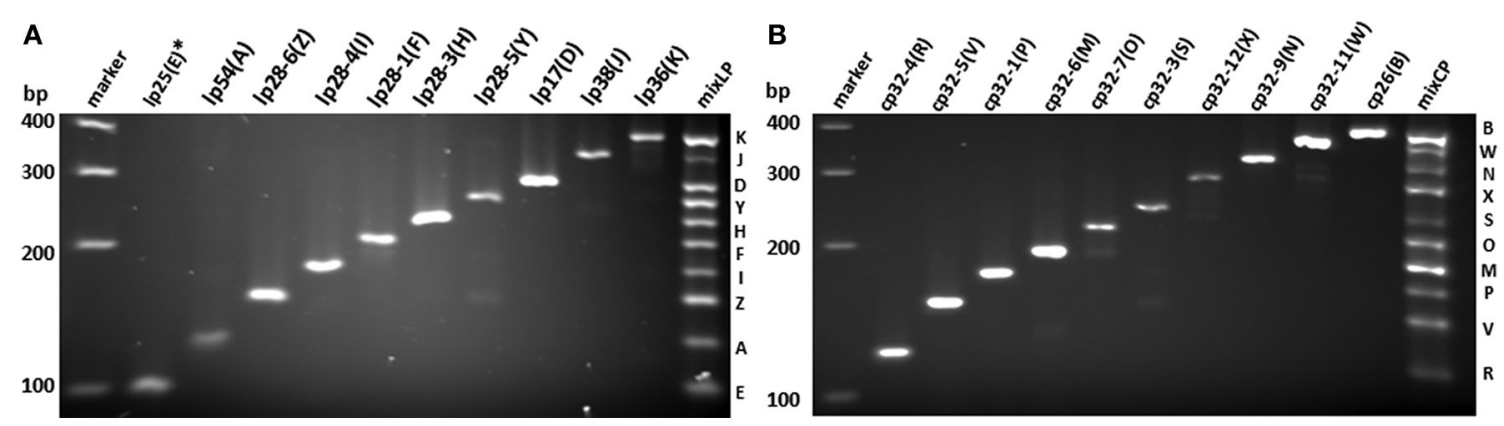

FIGURE 1 | Verification of PCR primer specificity and efficiency for each endogenous plasmid in $\boldsymbol{B}$. burgdorferi strain 297. PCR products were separated on 3\% metaphor agarose gel. Each product for linear (A) and circular (B) plasmids with corresponding designation were labeled on top. The mixed product from pooled primer mix for linear plasmids (mixLP) and circular plasmids (mixCP) were on the right of each gel, and the band corresponding to each plasmid is labeled with designated letter on the right. DNA ladder is labeled on the left. * The primer sequences for Ip25(E) were based on bptA sequence.

high level of $\mathrm{OspC}$ at 35 or $37^{\circ} \mathrm{C}$ at late logarithmic phase of growth (Yang et al., 2004; Gilbert et al., 2007; Ye et al., 2016a), even without performing the temperature shift from 23 to $35^{\circ} \mathrm{C}$, a condition often required for OspC induction in strain B31 (Schwan and Piesman, 2000). On the other hand, PL133 had relative low level of OspC (Figure 2A).

Given that certain gene(s) on endogenous plasmids can affect OspC levels of B. burgdorferi (Sarkar et al., 2011), it is possible that AH130 and PL133 may have different plasmid content when isolated from parental 297 strain, resulting in a phenotype of different OspC expression. Although AH130 and PL133 were thought to have a complete plasmid content, the previous work was done using B31 plasmid sequences since the 297 plasmid sequences was not available till recently. Upon examining the plasmid content, we found that AH130 and PL133 actually do not have a complete plasmid content: AH130 lost the linear plasmid lp28-5, whereas PL133 lost a circular plasmid cp32-3 (Figure 2B). These results suggest that (1) the parental B. burgdorferi strain 297 is a mixed clone; (2) the difference in plasmid contents may contribute to the difference in OspC production between AH130 and PL133.

\section{Absence of Ip28-5 is Not Associated with High OspC Level}

AH130 expressed very high level of OspC. One possibility is that $1 \mathrm{p} 28-5$ may encodes a gene that is inhibitory to ospC expression, and absence of 1 p28-5 leads to high OspC level. Given that the parental clone 297 contains mixed clones with OspC high or low phenotypes, one cannot draw conclusion from parental 297 that the presence of 1p28-5 can also have high OspC level. Thus, we investigated whether absence of lp28-5 leads to high OspC level by screening 297 clones isolated from semi-solid agar plate that lack $1 \mathrm{p} 28-5$, and identified three such clones (A-1, B-2, D-1) (Figure 3A). While two lp28-5-lacking clones (B-2, D-1) exhibited high OspC level, one clone (A1) had no detectable level of OspC on the Coomassie stained SDS gel (Figure 3B). This data suggests that absence of lp285 does not cause high level of ospC expression. Regarding the

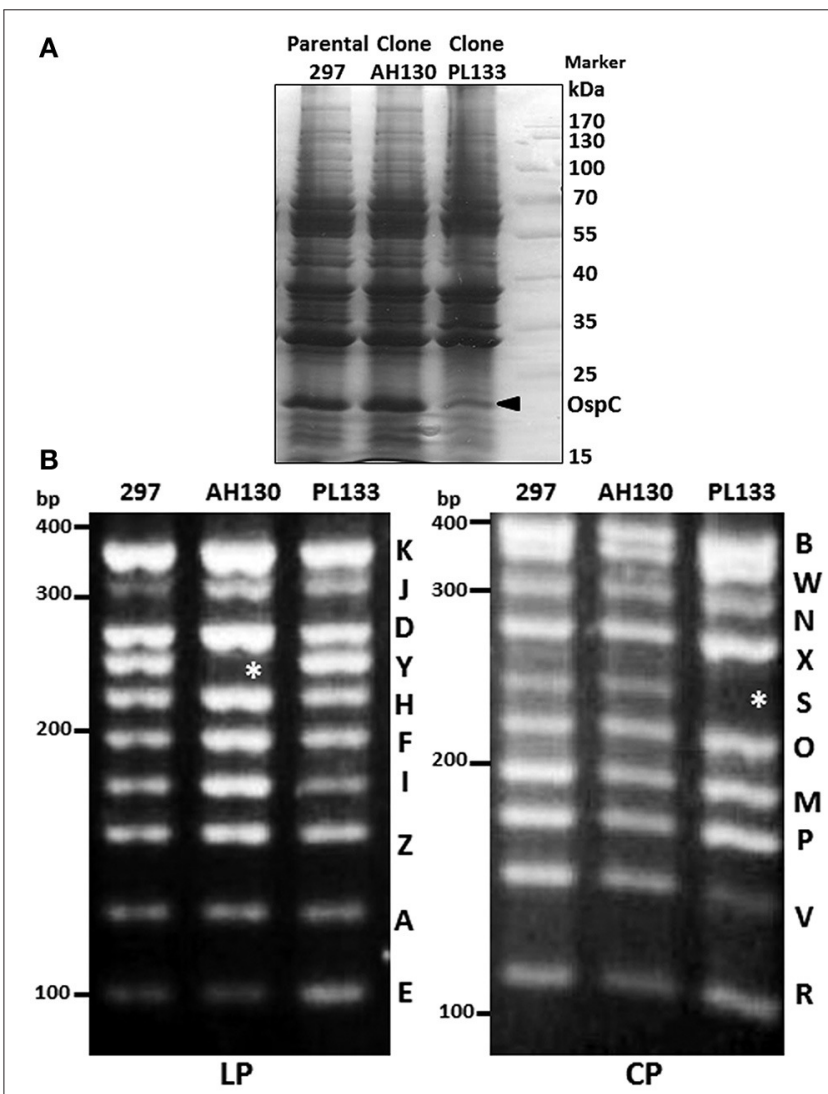

FIGURE 2 | Protein profile and plasmid content of $B$. burgdorferi strain 297 clones AH130 and PL133. (A) SDS-PAGE gel. B. burgdorferi strain 297 clones $\mathrm{AH} 130$ and $\mathrm{PL} 133$ were cultivated at $37^{\circ} \mathrm{C}$ and harvested at stationary phase of growth. The band corresponding to OspC is indicated by arrow. (B) Plasmid content of linear plasmid (LP) and circular plasmid (CP). Asterisk $\left(^{*}\right)$ indicates absent plasmids.

potential association between loss of cp32-3 and the low OspC level observed in PL133, we screened more than 50 clones to identify additional 297 clone lacking cp32-3 but were not successful. 


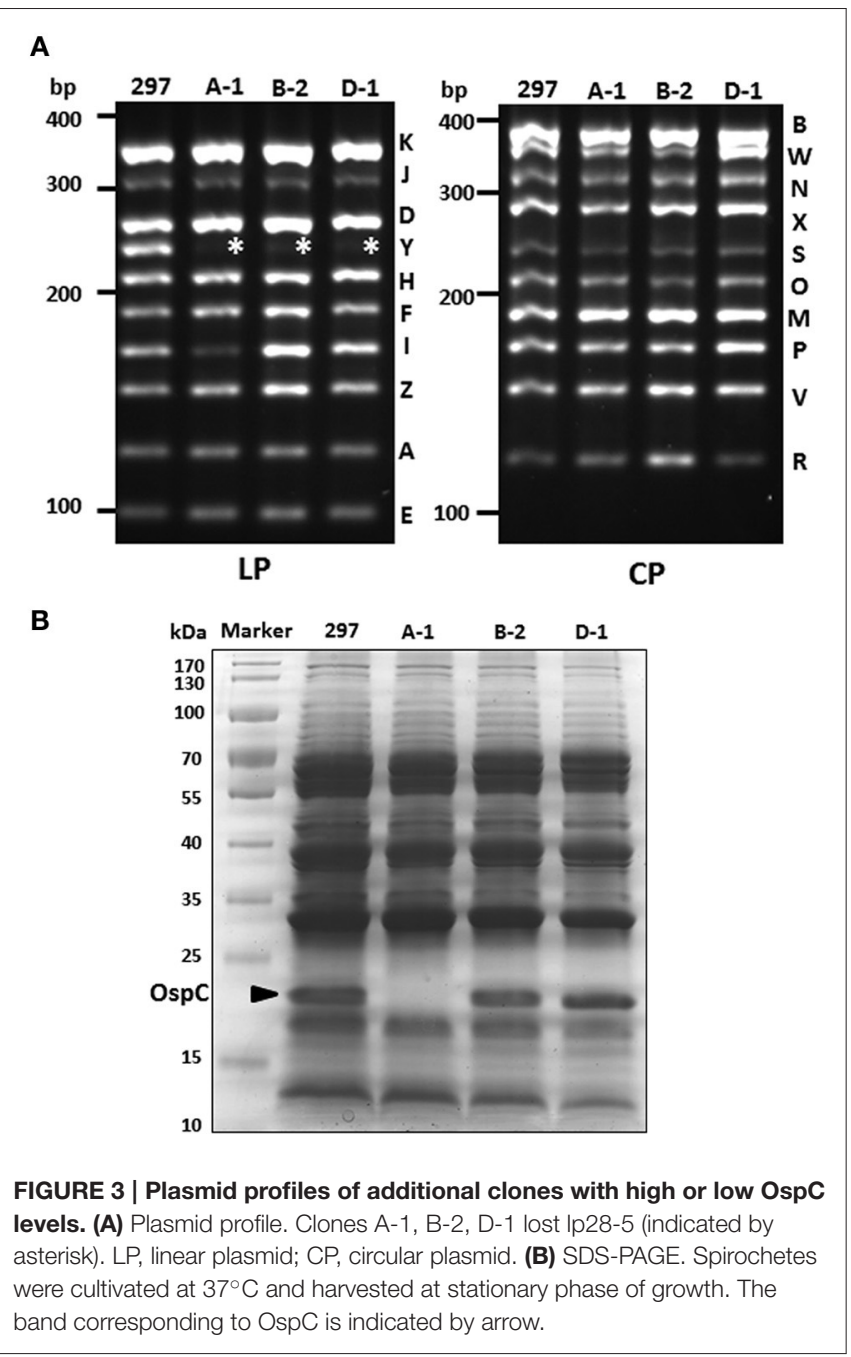

\section{Different Levels of RpoS and BosR among B. burgdorferi 297 Clones}

To further investigate what may contribute to the ospC expression variation among strain 297 clones, we examined the upstream regulators that govern $\operatorname{ospC}$ expression. $O s p C$ has a RpoS-type promoter which is controlled by RpoS (Hübner et al., 2001; Yang et al., 2005). As shown in Figures 4A,B, the low OspC clones, PL133 and A-1, had very low or undetectable levels of RpoS in comparison to high OspC clones (AH130, B-2, D-1). This result indicates that variation in OspC levels is due to the difference in RpoS levels in B. burgdorferi 297 clones.

Many factors can influence $r p o S$ and $o s p C$ expression such as Rrp2, RpoN, BosR, BadR, Rrp1, DsrA, BtmA, SodA, and BBD18 (Lybecker and Samuels, 2007; Hyde et al., 2009; Ouyang et al., 2009, 2011; Rogers et al., 2009; Miller et al., 2013; Sze et al., 2013; Troxell et al., 2013; Dulebohn et al., 2014; He et al., 2014; EsteveGassent et al., 2015; Ouyang and Zhou, 2015). In particular, expression of $r p o S$ is directly activated by the alternative factor RpoN as well as the transcriptional factor, BosR. Activation of the RpoN pathway is governed by phosphorylation of the

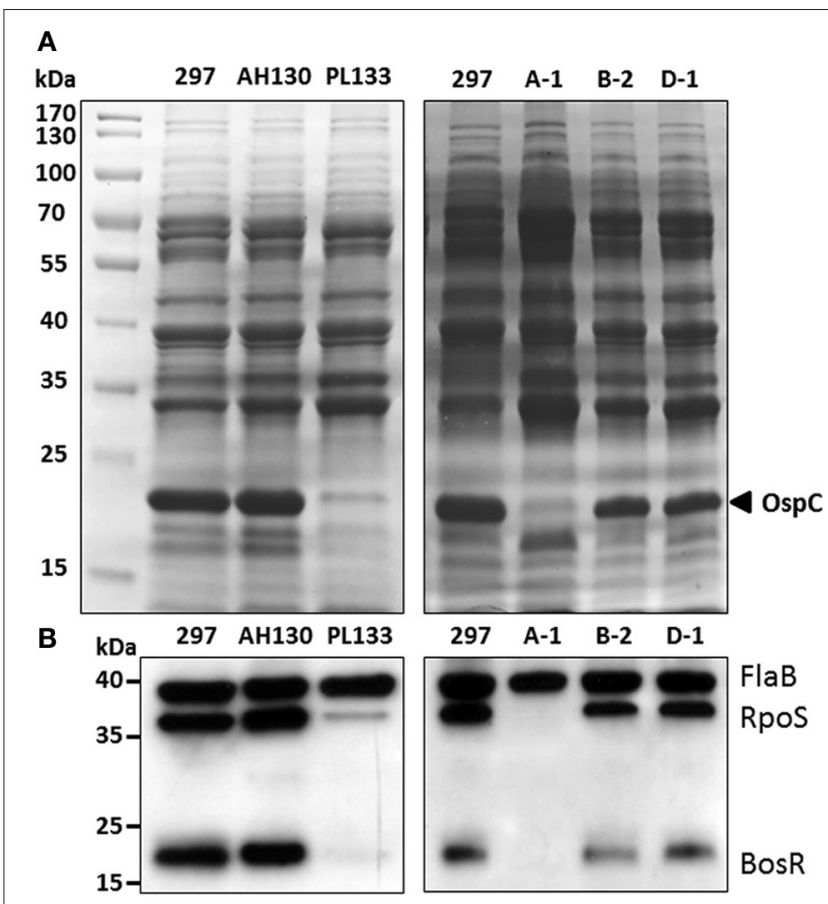

FIGURE 4 | Levels of RpoS and BosR in clones with different OspC levels. (A) SDS-PAGE. The band corresponding to OspC is labeled at the right. (B) Immunoblot. Whole cell lysates of $B$. burgdorferi clones were separated and probed with a mixture of $\alpha$-FlaB, $\alpha$-RpoS and $\alpha$-BosR monoclonal antibodies. FlaB serves as an internal control.

enhancer binding protein Rrp2 (Yang et al., 2003; Boardman et al., 2008). We recently showed that Rrp2 and RpoN is likely constitutively active under in vitro cultivation conditions, since phosphorylation of Rrp2 is vital for B. burgdorferi replication and Rrp2 is constitutively phosphorylated during in vitro replication (Groshong et al., 2012; Yin et al., 2016). Thus, Rrp2 is unlikely the factor that contributes to $o s p C$ expression variation among B. burgdorferi strains. Thus, we focused on the second factor, BosR. As shown in Figure 4B, PL133 and A-1 had very low or undetectable levels of BosR in comparison to high OspC clones (AH130, B-2, D-1). These results suggest that there is a difference in BosR levels among B. burgdorferi strains, which results in different levels of RpoS, and subsequently, ospC expression variation.

\section{DISCUSSION}

B. burgdorferi strain 297 is one of the widely used strain in borrelial research, especially for studying differential gene expression. Lack of a plasmid profiling method have made it difficult to track the plasmid contents of strain 297 during in vitro growth and genetic manipulation. Herein, we developed a multiplex PCR method that allows rapid plasmid profiling of strain 297. With such method, we found that the parental 297 strain is likely a mixed strain. Two of the commonly used infectious clones of strain 297, AH130 and PL133, which 
were previously thought having a complete endogenous plasmid content based on previous plasmid analysis using B31 plasmid sequences-derived primers, actually lost $1 \mathrm{p} 28-5$ and cp32-3, respectively. We further showed that the difference in plasmid profiles among $B$. burgdorferi clones could not explain ospC expression variation among the clones. On the other hand, we observed that there is a difference in BosR levels among the clones, which contributes to $o s p C$ expression variation.

Tracking each endogenous plasmid during every step of genetic manipulation of $B$. burgdorferi by PCR is timeconsuming. Previously, Bunikis et al., developed a multiplex PCR as a tool for validating plasmid profiling of B. burgdorferi strain B31, which has significantly improved the efficiency of the procedure of plasmid profiling (Bunikis et al., 2011). Unfortunately, the optimized primer pairs for plasmid profiling of strain B31 cannot be applied for other B. burgdorferi strains such as strain 297, due to sequence variation of endogenous plasmids among $B$. burgdorferi strains. The rapid plasmid profiling specifically for strain 297 reported in this study should be widely useful for borrelial research whenever B. burgdorferi 297 is used.

The data presented here shows that lp28-5 does not contribute to $o s p C$ expression variation among $B$. burgdorferi strains. Although we were unable to identify other clones missing cp32-3, the redundancy of cp32 plasmids in B. burgdorferi makes it unlikely that loss of cp32-3 is responsible for the variation in OspC expression. Thus, this study suggests that other yet-to-be identified genetic differences between the two strains contributes to OspC expression variation. On the other hand, this result does not conclude that endogenous plasmids do not play a role in regulation of $\operatorname{osp} C$ expression. In fact, $b b d 18$ on lp17 of $B$. burgdorferi strain B31 plays a negative role in regulation of rpoS and ospC expression (Sarkar et al., 2011; Dulebohn et al., 2014). The 297 strain also contains a similar lp17 plasmid. lp17 is one of the endogenous plasmids that are well-retained by B. burgdorferi under in vitro cultivation (Purser and Norris, 2000), so it is not surprising that we did not find a 297 clone lacking this plasmid.

The pattern of protein expression in the low OspC expressing clone A1 varied in different preparations and was sometimes significantly different from that seen in the high OspC expressing clones. For example, we noticed an increased intensity of a band below OspC in clone A1 in Figure 4A, but not in Figure 3. It has previously been proposed that downregulation of OspC is accompanied by upregulation of other lipoproteins ( $\mathrm{He}$ et al., 2008; Xu et al., 2008). It is noteworthy that different clones

\section{REFERENCES}

Barbour, A. G. (1984). Isolation and cultivation of Lyme disease spirochetes. Yale J. Biol. Med. 57, 521-525.

Blevins, J. S., Revel, A. T., Smith, A. H., Bachlani, G. N., and Norgard, M. V. (2007). Adaptation of a luciferase gene reporter and lac expression system to Borrelia burgdorferi. Appl. Environ. Microbiol. 73, 1501-1513. doi: 10.1128/AEM.02454-06

Boardman, B. K., He, M., Ouyang, Z., Xu, H., Pang, X., and Yang, X. F. (2008). Essential role of the response regulator Rrp2 in the infectious cycle of Borrelia burgdorferi. Infect. Immun. 76, 3844-3853. doi: 10.1128/IAI.00467-08 derived from isolates of $B$. burgdorferi strain B31 have been shown to have considerable heterogeneity not only in protein profile and plasmid content, but also in colony phenotype on solid agar (Elias et al., 2002). We screened and compared strain 297 clones with different colony morphologies, and found that colony morphology is not associated with plasmid content (data not shown).

In addition, B. burgdorferi strain B31 presents different types of colony morphology on the semi-solid agar plate. Thus, we also screened and compared groups with different colony morphologies, and found that colony morphology is not associated with plasmid content (data not shown).

BosR is a FurR/PerR homolog (for review, see, Samuels and Radolf, 2009). It is required for activation of rpoS expression in B. burgdorferi, but the mechanism remains unknown. Recently, it was reported that BosR undergoes autoregulation (Ouyang et al., 2016). However, regulation of bosR expression in response to environmental signals by large remains unknown. The study herein demonstrates that the BosR levels are highly variable among $B$. burgdorferi strains. Continuing efforts on the investigation of clonal differences will likely help uncover the mechanism of regulation of BosR by environmental factors.

\section{ETHICS STATEMENT}

The work conducted in the manuscript was approved by Indiana University School of Medicine Biosafety Committee (protocol number 643).

\section{AUTHOR CONTRIBUTIONS}

XX, YY, XY, YL conceived and designed the experiments; XX, JD, and TL performed the experiments; XX and TC analyzed the data; XX, YL, and XY wrote the paper. XX and YY contributed equally to this work.

\section{ACKNOWLEDGMENTS}

Fundings for this work were partially provided by $\mathrm{NIH}$ grants AI083640 and AI085242 (to XY), and the National Science Foundation of China 81428015, 81171611 (YL). This investigation was partially conducted in a facility with support from research facilities improvement program grant number C06 RR015481-01 from the National Center for Research Resources, NIH.
Bunikis, I., Kutschan-Bunikis, S., Bonde, M., and Bergström, S. (2011). Multiplex PCR as a tool for validating plasmid content of Borrelia burgdorferi. J. Microbiol. Methods 86, 243-247. doi: 10.1016/j.mimet.2011.05.004

Burgdorfer, W., Barbour, A., Hayes, S., Benach, J., Grunwaldt, E., and Davis, J. (1982). Lyme disease-a tick-borne spirochetosis? Science 18, 1317-1319. doi: 10.1126/science.7043737

Caimano, M. J., Drecktrah, D., Kung, F., and Samuels, D. S. (2016). Interaction of the Lyme disease spirochete with its tick vector. Cell. Microbiol. 18, 919-927. doi: $10.1111 / \mathrm{cmi} .12609$

Caimano, M. J., Yang, X., Popova, T. G., Clawson, J. L., Akins, D. R., Norgard, M. V., et al. (2000). Molecular and evolutionary characterization of the cp32/18 
family of supercoiled plasmids in Borrelia burgdorferi 297. Infect. Immun. 68, 1574-1586. doi: 10.1128/IAI.68.3.1574-1586.2000

Carrasco, S. E., Troxell, B., Yang, Y., Brandt, S. L., Li, H., Sandusky, G. E., et al. (2015a). Outer Surface Protein OspC is an antiphagocytic factor that protects Borrelia burgdorferi from phagocytosis by macrophages. Infect. Immun. 83, 4848-4860. doi: 10.1128/IAI.01215-15

Carrasco, S. E., Yang, Y., Troxell, B., Yang, X., Pal, U., and Yang, X. F. (2015b). Borrelia burgdorferi elongation factor EF-Tu is an immunogenic protein during Lyme borreliosis. Emerg. Microbes Infect. 4:e54. doi: 10.1038/emi.2015.54

Casjens, S. R., Mongodin, E. F., Qiu, W.-G., Luft, B. J., Schutzer, S. E., Gilcrease, E. B., et al. (2012). Genome stability of Lyme disease spirochetes: comparative genomics of Borrelia burgdorferi plasmids. PLoS ONE 7:e33280. doi: 10.1371/journal.pone.0033280

Casjens, S., Palmer, N., van Vugt, R., Huang, W. M., Stevenson, B., Rosa, P., et al. (2000). A bacterial genome in flux: the twelve linear and nine circular extrachromosomal DNAs in an infectious isolate of the Lyme disease spirochete Borrelia burgdorferi. Mol. Microbiol. 35, 490-516. doi: 10.1046/j.1365-2958.2000.01698.x

Chaconas, G., and Norris, S. J. (2013). Peaceful coexistence amongst Borrelia plasmids: getting by with a little help from their friends? Plasmid 70, 161-167. doi: 10.1016/j.plasmid.2013.05.002

Dulebohn, D. P., Hayes, B. M., and Rosa, P. A. (2014). Global repression of hostassociated genes of the lyme disease spirochete through post-transcriptional modulation of the alternative sigma factor RpoS. PLoS ONE 9:e93141. doi: 10.1371/journal.pone.0093141

Elias, A. F., Stewart, P. E., Grimm, D., Caimano, M. J., Eggers, C. H., Tilly, K., et al. (2002). Clonal polymorphism of Borrelia burgdorferi strain B31 MI: implications for mutagenesis in an infectious strain background. Infect. Immun. 70, 2139-2150. doi: 10.1128/IAI.70.4.2139-2150.2002

Esteve-Gassent, M. D., Smith, T. C. II, Small, C. M., Thomas, D. P., and Seshu, J. (2015). Absence of sodA increases the levels of oxidation of key metabolic determinants of Borrelia burgdorferi. PLOS ONE 10:e0136707. doi: 10.1371/journal.pone. 0136707

Fraser, C. M., Casjens, S., Huang, W. M., Sutton, G. G., Clayton, R., Lathigra, R., et al. (1997). Genomic sequence of a Lyme disease spirochaete, Borrelia burgdorferi. Nature 390, 580-586. doi: 10.1038/37551

Gilbert, M. A., Morton, E. A., Bundle, S. F., and Samuels, D. S. (2007). Artificial regulation of ospC expression in Borrelia burgdorferi. Mol. Microbiol. 63, 1259-1273. doi: $10.1111 /$ j.1365-2958.2007.05593.x

Grimm, D., Eggers, C. H., Caimano, M. J., Tilly, K., Stewart, P. E., Elias, A. F., et al. (2004a). Experimental assessment of the roles of linear plasmids lp25 and lp281 of Borrelia burgdorferi throughout the infectious cycle. Infect. Immun. 72, 5938-5946. doi: 10.1128/IAI.72.10.5938-5946.2004

Grimm, D., Tilly, K., Byram, R., Stewart, P. E., Krum, J. G., Bueschel, D. M., et al. (2004b). Outer-surface protein C of the Lyme disease spirochete: a protein induced in ticks for infection of mammals. Proc. Natl. Acad. Sci. U.S.A. 101, 3142-3147. doi: 10.1073/pnas.0306845101

Groshong, A. M., Gibbons, N. E., Yang, X. F., and Blevins, J. S. (2012). Rrp2, a prokaryotic enhancer-like binding protein, is essential for viability of Borrelia burgdorferi. J. Bacteriol. 194, 3336-3342. doi: 10.1128/JB.00253-12

He, M., Oman, T., Xu, H., Blevins, J. S., Norgard, M. V., and Yang, X. F. (2008). Abrogation of ospAB constitutively activates the Rrp2-RpoN-RpoS pathway (SigmaN-SigmaS Cascade) in Borrelia burgdorferi. Mol. Microbiol. 70, 1453-1464. doi: 10.1111/j.1365-2958.2008.06491.x

He, M., Zhang, J.-J., Ye, M., Lou, Y., and Yang, X. F. (2014). The cyclic di-GMP receptor PlzA controls virulence gene expression through RpoS in Borrelia burgdorferi. Infect. Immun. 82, 445-452. doi: 10.1128/IAI.01238-13

Hübner, A., Yang, X., Nolen, D. M., Popova, T. G., Cabello, F. C., and Norgard, M. V. (2001). Expression of Borrelia burgdorferi OspC and DbpA is controlled by a RpoN-RpoS regulatory pathway. Proc. Natl. Acad. Sci. U.S.A. 98, 12724-12729. doi: $10.1073 /$ pnas.231442498

Hughes, C. N., Kodner, C. B., and Johnson, R. C. (1992). DNA analysis of Borrelia burgdorferi NCH-1, the first northcentral U.S. human Lyme disease isolate. J. Clin. Microbiol. 30, 698-703.

Hyde, J. A., Shaw, D. K., Smith Iii, R., Trzeciakowski, J. P., and Skare, J. T. (2009). The BosR regulatory protein of Borrelia burgdorferi interfaces with the RpoS regulatory pathway and modulates both the oxidative stress response and pathogenic properties of the Lyme disease spirochete. Mol. Microbiol. 74, 1344-1355. doi: 10.1111/j.1365-2958.2009.06951.x

Lybecker, M. C., and Samuels, D. S. (2007). Temperature-induced regulation of RpoS by a small RNA in Borrelia burgdorferi. Mol. Microbiol. 64, 1075-1089. doi: 10.1111/j.1365-2958.2007.05716.x

Miller, C. L., Karna, S. L., and Seshu, J. (2013). Borrelia host adaptation regulator (BadR) regulates rpoS to modulate host adaptation and virulence factors in Borrelia burgdorferi. Mol. Microbiol. 88, 105-124. doi: 10.1111/mmi.12171

Ouyang, Z., and Zhou, J. (2015). BadR (BB0693) controls growth phase-dependent induction of rpoS and bosR in Borrelia burgdorferi via recognizing TAAAATAT motifs. Mol. Microbiol. 98, 1147-1167. doi: 10.1111/mmi.13206

Ouyang, Z., Deka, R. K., and Norgard, M. V. (2011). BosR (BB0647) controls the RpoN-RpoS regulatory pathway and virulence expression in Borrelia burgdorferi by a novel DNA-binding mechanism. PLoS Pathog. 7:e1001272. doi: 10.1371/journal.ppat.1001272

Ouyang, Z., Kumar, M., Kariu, T., Haq, S., Goldberg, M., Pal, U., et al. (2009). BosR (BB0647) governs virulence expression in Borrelia burgdorferi. Mol. Microbiol. 74, 1331-1343. doi: 10.1111/j.1365-2958.2009.06945.x

Ouyang, Z., Zhou, J., and Norgard, M. V. (2014). Synthesis of RpoS Is dependent on a putative enhancer binding protein Rrp2 in Borrelia burgdorferi. PLoS ONE 9:e96917. doi: 10.1371/journal.pone.0096917

Ouyang, Z., Zhou, J., and Norgard, M. V. (2016). Evidence that BosR (BB0647) is a positive autoregulator in Borrelia burgdorferi. Infect. Immun. 84, 2566-2574. doi: 10.1128/IAI.00297-16

Pal, U., Yang, X., Chen, M., Bockenstedt, L. K., Anderson, J. F., Flavell, R. A., et al. (2004). OspC facilitates Borrelia burgdorferi invasion of Ixodes scapularis salivary glands. J. Clin. Invest. 113, 220-230. doi: 10.1172/JCI200419894

Purser, J. E., and Norris, S. J. (2000). Correlation between plasmid content and infectivity in Borrelia burgdorferi. Proc. Natl. Acad. Sci. U.S.A. 97, 13865-13870. doi: 10.1073/pnas.97.25.13865

Purser, J. E., Lawrenz, M. B., Caimano, M. J., Howell, J. K., Radolf, J. D., and Norris, S. J. (2003). A plasmid-encoded nicotinamidase (PncA) is essential for infectivity of Borrelia burgdorferi in a mammalian host. Mol. Microbiol. 48, 753-764. doi: 10.1046/j.1365-2958.2003.03452.x

Radolf, J. D., Caimano, M. J., Stevenson, B., and Hu, L. T. (2012). Of ticks, mice and men: understanding the dual-host lifestyle of Lyme disease spirochaetes. Nat. Rev. Microbiol. 10, 87-99. doi: 10.1038/nrmicro2714

Revel, A. T., Blevins, J. S., Almazán, C., Neil, L., Kocan, K. M., de la Fuente, J., et al. (2005). bptA (bbe16) is essential for the persistence of the Lyme disease spirochete, Borrelia burgdorferi, in its natural tick vector. Proc. Natl. Acad. Sci. U.S.A. 102, 6972-6977. doi: 10.1073/pnas.0502565102

Rogers, E. A., Terekhova, D., Zhang, H., Hovis, K. M., Schwartz, I., and Marconi, R. T. (2009). Rrp1, a cyclic-di-GMP-producing response regulator, is an important regulator of Borrelia burgdorferi core cellular functions. Mol. Microbiol. 71, 1551-1573. doi: 10.1111/j.1365-2958.2009.06621.x

Samuels, D. S. (1995). "Electrotransformation of the spirochete Borrelia burgdorferi," in Methods in Molecular Biology, ed J. A. Nickoloff (Totowa, NJ: Humana Press), 253-259.

Samuels, D. S. (2011). Gene regulation in Borrelia burgdorferi. Annu. Rev. Microbiol. 65, 479-499. doi: 10.1146/annurev.micro.112408.134040

Samuels, D. S., and Radolf, J. D. (2009). Who is the BosR around here anyway? Mol. Microbiol. 74, 1295-1299. doi: 10.1111/j.1365-2958.2009.06971.x

Sarkar, A., Hayes, B. M., Dulebohn, D. P., and Rosa, P. A. (2011). Regulation of the virulence determinant OspC by bbd18 on linear plasmid lp17 of Borrelia burgdorferi. J. Bacteriol. 193, 5365-5373. doi: 10.1128/JB.01496-10

Schutzer, S. E., Fraser-Liggett, C. M., Casjens, S. R., Qiu, W.-G., Dunn, J. J., Mongodin, E. F., et al. (2011). Whole-genome sequences of thirteen isolates of Borrelia burgdorferi. J. Bacteriol. 193, 1018-1020. doi: 10.1128/JB.0 $1158-10$

Schwan, T. G., and Piesman, J. (2000). Temporal changes in outer surface proteins $\mathrm{A}$ and $\mathrm{C}$ of the Lyme disease-associated spirochete, Borrelia burgdorferi, during the chain of infection in ticks and mice. J. Clin. Microbiol. 38, 382-388.

Steere, A. C., Grodzicki, R. L., Kornblatt, A. N., Craft, J. E., Barbour, A. G., Burgdorfer, W., et al. (1983). The spirochetal etiology of Lyme disease. N. Engl. J. Med. 308, 733-740. doi: 10.1056/NEJM198303313081301

Sze, C. W., Smith, A., Choi, Y. H., Yang, X., Pal, U., Yu, A., et al. (2013). Study of the response regulator Rrp1 reveals its regulatory role in chitobiose 
utilization and virulence of Borrelia burgdorferi. Infect. Immun. 81, 1775-1787. doi: 10.1128/IAI.00050-13

Troxell, B., Ye, M., Yang, Y., Carrasco, S. E., Lou, Y., and Yang, X. F. (2013). Manganese and zinc regulate virulence determinants in Borrelia burgdorferi. Infect. Immun. 81, 2743-2752. doi: 10.1128/IAI.00507-13

Xu, H. J., He, M., He, J. J., and Yang, X. F. (2010). Role of the surface lipoprotein BBA07 in the enzootic cycle of Borrelia burgdorferi. Infect. Immun. 78, 2910-2918. doi: 10.1128/IAI.00372-10

Xu, Q., McShan, K., and Liang, F. T. (2008). Essential protective role attributed to the surface lipoproteins of Borrelia burgdorferi against innate defences. Mol. Microbiol. 69, 15-26. doi: 10.1111/j.1365-2958.2008.06264.x

Yang, X. F., Alani, S. M., and Norgard, M. V. (2003). The response regulator Rrp2 is essential for the expression of major membrane lipoproteins in Borrelia burgdorferi. Proc. Natl. Acad. Sci. U.S.A. 100, 11001-11006. doi: 10.1073/pnas. 1834315100

Yang, X. F., Lybecker, M. C., Pal, U., Alani, S. M., Blevins, J., Revel, A. T., et al. (2005). Analysis of the $\operatorname{sp} C$ regulatory element controlled by the RpoNRpoS regulatory pathway in Borrelia burgdorferi. J. Bacteriol. 187, 4822-4829. doi: 10.1128/JB.187.14.4822-4829.2005

Yang, X. F., Pal, U., Alani, S. M., Fikrig, E., and Norgard, M. V. (2004). Essential role for OspA/B in the life cycle of the Lyme disease spirochete. J. Exp. Med. 199, 641-648. doi: 10.1084/jem.20031960

Yang, X., Goldberg, M. S., Popova, T. G., Schoeler, G. B., Wikel, S. K., Hagman, K. E., et al. (2000). Interdependence of environmental factors influencing reciprocal patterns of gene expression in virulent Borrelia burgdorferi. Mol. Microbiol. 37, 1470-1479. doi: 10.1046/j.1365-2958.2000.02104.x

Ye, M., Sharma, K., Thakur, M., Smith, A. A., Buyuktanir, O., Xiang, X., et al. (2016a). BbHtrA, a temperature- and stationary phase-activated protease involved in maturation of a key microbial virulence determinant, facilitates Borrelia burgdorferi infection in mammalian hosts. Infect. Immun. 84, 2372-2381. doi: 10.1128/IAI.00360-16

Ye, M., Zhou, Y., Lou, Y., and Yang, X. F. (2016b). Genome reduction of Borrelia burgdorferi: two TCS signaling pathways for two distinct host habitats. Sci. China Life Sci. 59, 19. doi: 10.1007/s11427-015-4996-Z

Yin, Y., Yang, Y., Xiang, X., Wang, Q., Yang, Z.-N., Blevins, J., et al. (2016). Insight into the dual functions of bacterial enhancer-binding protein Rrp2 of Borrelia burgdorferi. J. Bacteriol. 198, 1543-1552. doi: 10.1128/JB.01010-15

Conflict of Interest Statement: The authors declare that the research was conducted in the absence of any commercial or financial relationships that could be construed as a potential conflict of interest.

Copyright $\odot 2017$ Xiang, Yang, Du, Lin, Chen, Yang and Lou. This is an open-access article distributed under the terms of the Creative Commons Attribution License (CC $B Y$ ). The use, distribution or reproduction in other forums is permitted, provided the original author(s) or licensor are credited and that the original publication in this journal is cited, in accordance with accepted academic practice. No use, distribution or reproduction is permitted which does not comply with these terms. 